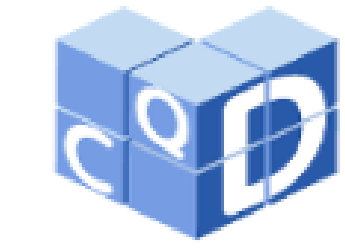

Revista Eletrônica

Paulista de Matemática

ISSN 2316-9664

Volume 18, jul. 2020

Iniciação Científica

Reginaldo Leoncio Silva Universidade Estadual do Sudoeste da Bahia - UESB

reggekant@yahoo.com.br

Roger Luiz da Silva Almeida Universidade Estadual do Sudoeste da Bahia - UESB rogerluizzz@bol.com.br

\section{A fantástica sequência de Fibonacci e o enigmático número de ouro: contexto histórico, definições, propriedades e aplicações}

The fantastic Fibonacci sequence and the enigmatic gold number: historical context, definitions, properties and applications

\section{Resumo}

O conteúdo sobre números de Fibonacci e razão áurea é uma oportunidade riquíssima para o professor trabalhar a conexão da Matemática com o dia-a-dia. Assim, neste trabalho falaremos sobre estes assuntos e destacaremos suas aplicações práticas. Começaremos falando um pouco do contexto histórico, destacando aspectos sobre a vida de Fibonacci e suas obras, depois abordaremos o estudo formal de sua sequência, definindo-a e destacando algumas de suas principais propriedades. Em seguida, abordaremos sobre o número de ouro com sua definição e um pouco do contexto histórico. Depois falaremos sobre o retângulo áureo e a espiral áurea. Em seguida mostraremos algumas curiosidades desse número e destacaremos a sua bela relação com a sequência de Fibonacci. Finalmente, evidenciaremos algumas aplicações de tal sequência e do número dourado.

Palavras-chave: Sequência de Fibonacci, Número de ouro, Espiral áurea, Aplicações

\begin{abstract}
The content on Fibonacci numbers and golden ratio is a very rich opportunity for the teacher to work on the connection between mathematics and everyday life. Thus, in this work we will talk about these subjects and highlight their practical applications. We will start by talking a little about the historical context, highlighting aspects of Fibonacci's life and his works, then we will approach the formal study of its sequence, defining it and highlighting some of its main properties. Then, we will approach the gold number with its definition and a little of the historical context. Then we will talk about the golden rectangle and the golden spiral. Next, we will show you some curiosities from that issue and highlight its beautiful relationship with the Fibonacci sequence. Finally, we will highlight some applications of such a sequence and the golden number
\end{abstract}

Keywords: Fibonacci sequence, Gold number; Golden spiral, Applications. 


\section{Introdução}

Sabemos que a Matemática é uma Ciência muito interessante e há muitas coisas fascinantes para se estudar. Ela é uma Ciência muito especial e é uma ferramenta importantíssima em muitas situações práticas. Ao contrário do que muitos pensam, ela também está muito relacionada com o mundo natural, como bem enfatiza Contador (2011).

Um dos assuntos da Matemática que é de extrema beleza e riqueza é, certamente, a sequência de Fibonacci e o número de ouro. Com relação a este último sabe-se que é um número enigmático conhecido desde a Antiguidade. O mesmo aparece nas pirâmides do Egito, nos Elementos de Euclides, nas obras dos Pitagóricos, etc. O número de ouro inspirou o trabalho de muitos artistas, dentre os quais podemos citar Leonardo da Vinci, que é o autor da famosa obra Monalisa. Já a sequência de Fibonacci é advinda do problema de reprodução dos coelhos de Leonardo de Pisa. Esta sequência foi posteriormente estudada por muitos outros matemáticos, que descobriram inúmeras propriedades interessantes. O que é mais intrigante sobre tal sequência é sua relação com o número de ouro, dando origem a uma curva conhecida como espiral áurea.

A sequência de Fibonacci e o número dourado estão intimamente ligados com o mundo concreto. Eles aparecem em muitas situações da natureza: em árvores, plantas, animais, etc. Estas aparições são com a sequência propriamente dita, com a razão áurea ou com a espiral áurea, como veremos em frente.

\section{Contexto histórico}

Nesta seção falaremos um pouco sobre a vida de Fibonacci, elucidando algumas de suas obras. Além disso, apresentaremos o problema que deu origem a sua sequência.

\subsection{Biografia e obras de Fibonacci}

Leonardo de Pisa era considerado o mais notável matemático da idade média. O ano de seu nascimento é um ponto de discórdia para alguns autores: de acordo com Contador (2011) e Eves (2004), o mesmo nasceu em 1175 e morreu em 1250. Já para Boyer (1974), ele viveu de 1180 a 1250. Leonardo era filho de um comerciante italiano chamado "Guilielmo Bonacci", por isso ficou conhecido como Fibonacci. Iniciou estudando assuntos relacionados a negócios e comércio mercantil, recebendo parte de sua educação em Bejaia, norte da África, onde seu pai desempenhava uma função alfandegária. Fibonacci teve a oportunidade de conhecer e estudar o sistema de numeração indo-arábico, adotando-o e tornando-se um defensor. Ele considerava que este sistema era mais simples e eficiente do que os algarismos romanos.

De volta à Itália Leonardo Fibonacci escreveu, vários livros: Liber Abaci (1202), Practica Geometriae (1220), Flos (1225) e Liber Quadratorum (1225).

\subsection{O problema de reprodução dos coelhos}

De todos os temas e problemas tratados no Liber Abaci, o que mais se destacou e que ainda hoje cria-se novas aplicações é o problema dos coelhos, elucidado por Boyer (1974, p. 186): "Quantos pares de coelhos são produzidos num ano, começando com um só par, se em cada mês gera um novo par que se torna produtivo a partir do segundo mês?"

\section{Solução do problema:}


A solução desse problema gera uma sequência que é amplamente estudada com várias aplicações na natureza e recheada de inúmeras propriedades interessantes, como veremos mais adiante. Ela é conhecida como sequência de Fibonacci. Veja na figura abaixo:

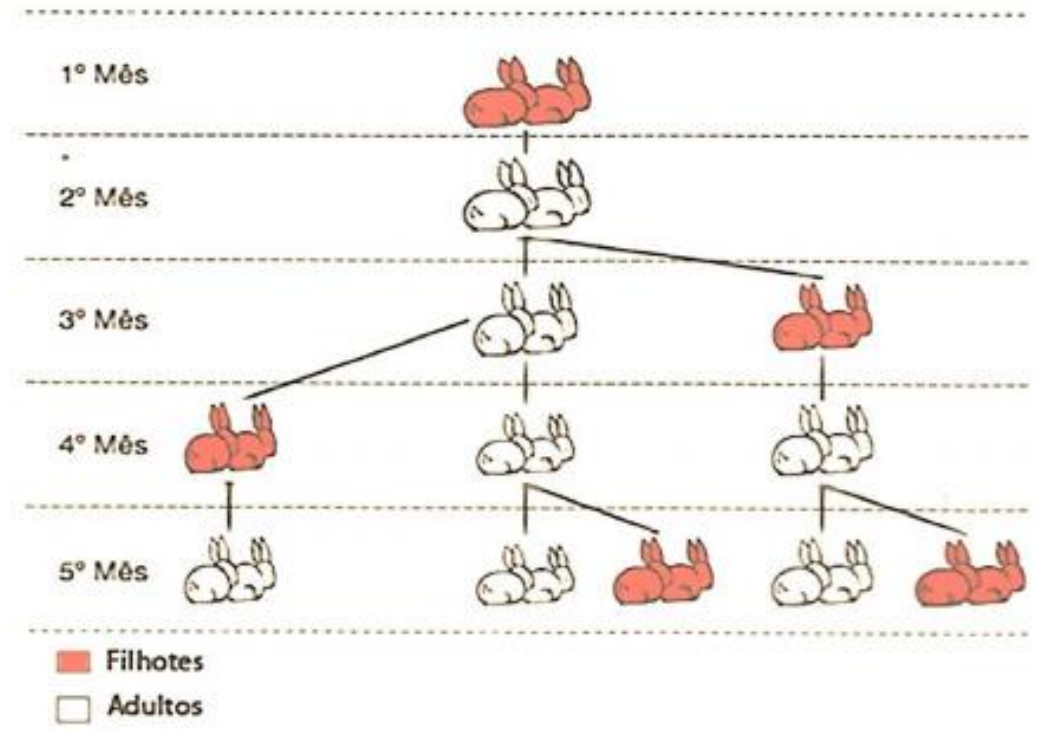

Figura 1- Esquema de reprodução dos coelhos Fonte: Silva (2014, pg. 23)

\section{A sequência de Fibonacci}

Nesta seção iremos definir a sequência de Fibonacci, evidenciando algumas de suas propriedades.

\subsection{Definição da sequência}

Definição: A sequência de inteiros positivos $\left(F_{n}\right):(1,1,2,3,5,8,13,21, \ldots)$, onde $F_{1}=$ $F_{2}=1$ e $F_{n}=F_{n-1}+F_{n-2}$, para todo $n>2, n \in \mathbb{N}$, recebe o nome de sequência de Fibonacci. Os seus termos chamam-se números de Fibonacci.

\subsection{Propriedades elementares}

A sequência de Fibonacci é repleta de propriedades muito interessantes. Abaixo segue algumas de suas propriedades elementares:

Propriedade 1: A soma dos $n$ primeiros números de Fibonacci é igual a $F_{n+2}-1$.

Prova:

De fato, temos que:

$F_{1}=F_{3}-F_{2}=F_{3}-1$

$F_{2}=F_{4}-F_{3}$

$F_{3}=F_{5}-F_{4}$

$F_{n-1}=F_{n+1}-F_{n}$ 


$$
F_{n}=F_{n+2}-F_{n+1}
$$

Somando membro a membro as igualdades e simplificando, temos que: $\sum_{i=1}^{n} F_{i}=$ $F_{n+2}-1$.

Propriedade 2: A soma dos $n$ primeiros números de Fibonacci com índices impares é igual a $F_{2 n}$.

Prova: É análoga a propriedade 1.

Propriedade 3: A soma dos $n$ primeiros números de Fibonacci com índices pares é igual a $F_{2 n+1}-1$.

Prova: É análoga a propriedade 1.

Propriedade 4: Quaisquer dois números de Fibonacci consecutivos são primos entre si.

\section{Prova:}

Vamos provar usando o princípio de indução matemática sobre $n$. Para maiores detalhes sobre este princípio, o leitor pode procurar Oliveira e Fernández (2010).

Seja $P(n)$ a seguinte proposição: $m d c\left(F_{n}, F_{n+1}\right)=1, n \geq 1$. Para $n=1$ a afirmação é trivialmente verificada, pois $\operatorname{mdc}(1,2)=1$. Suponhamos que $\operatorname{mdc}\left(F_{n}, F_{n+1}\right)=1, n \geq 1$, mostraremos que $\operatorname{mdc}\left(F_{n+1}, F_{n+2}\right)=1$.

Usando a hipótese de indução, temos que:

$\operatorname{mdc}\left(F_{n+1}, F_{n+2}\right)=\operatorname{mdc}\left(F_{n+1}, F_{n}+F_{n+1}\right)=\operatorname{mdc}\left(F_{n+1}, F_{n}+F_{n+1}-F_{n+1}\right)=$ $\operatorname{mdc}\left(F_{n+1}, F_{n}\right)=\operatorname{mdc}\left(F_{n}, F_{n+1}\right)=1$, como queríamos provar.

\section{0 número de ouro}

Nesta seção iremos apresentar o número de ouro, a razão áurea, o retângulo e a espiral áurea, além de mostrarmos a bela relação entre o número de ouro e a sequência de Fibonacci.

\subsection{Definição do número de ouro}

Definição: O número de ouro, também conhecido como proporção áurea, número áureo, secção áurea, proporção de ouro, é um número irracional representado pela letra grega $\emptyset(p h i)$, cujo valor é:

$$
\varnothing=\frac{1+\sqrt{5}}{2}
$$

Este número, que é muito misterioso e enigmático, pode ser identificado em muitas situações concretas, como por exemplo, na natureza, na música, na arte e nas grandes construções feitas pelos homens.

\subsection{A seção áurea}

Definição: Diz-se que um ponto divide um segmento de reta em média e extrema razão ou em seção áurea, se o mais longo dos segmentos é média geométrica entre o menor e o segmento todo. A razão entre o maior segmento e o menor segmento chama-se razão áurea.

Entre outras palavras, dado um segmento $A B$ de medida $x+y$, seja $C$ o ponto entre $A$ e $B$, tal que $A C>C B$, como mostra a figura abaixo:

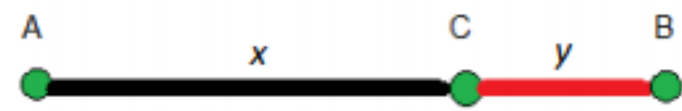


Figura 2 - Segmento dividido na razão áurea

Assim, temos que:

$\frac{A C}{B C}=\frac{A B}{A C}$, ou seja,

$\frac{x}{y}=\frac{x+y}{x} \rightarrow x^{2}-x y-y^{2}=0$

Dividindo ambos os membros por $y^{2}$, temos que:

$\left(\frac{x}{y}\right)^{2}-\frac{x}{y}-1=0$

Como $\varnothing=\frac{x}{y}$, resulta que $\emptyset^{2}-\varnothing-1=0$.

Resolvendo esta última equação, obtemos: $\emptyset=\frac{1 \pm \sqrt{5}}{2}$.

Como $\emptyset>0$, concluímos que $\varnothing=\frac{1+\sqrt{5}}{2}$.

\subsection{O retângulo áureo, a sequência de Fibonacci e a espiral áurea}

Definição: O retângulo áureo é o retângulo no qual a razão entre as medidas de seus lados é o número de ouro, ou seja, se $x$ e $y$ são, respectivamente, o maior e o menor lado, tem se que:

$$
\frac{x}{y}=\varnothing=\frac{1+\sqrt{5}}{2}
$$

Por ser considerado uma figura esteticamente agradável, este retângulo exerceu enorme influência em obras arquitetônicas e em pinturas. O que existe de mais fascinante com o retângulo áureo, é sua relação com a sequência de Fibonacci, dando origem a chamada espiral áurea, como mostraremos logo abaixo.

\subsubsection{Construção da espiral áurea}

Tomemos um quadrado de lado $l=1$. Agora coloquemos dois destes quadrados, um sobre o outro, obtendo um retângulo de lados 1 e 2. A partir do lado maior construímos um quadrado de lado 2 na parte esquerda dos quadrados de lado 1 . Note que os dois quadrados de lado 1 junto com o de lado 2 formam um retângulo de lado 3 . A partir deste lado tracemos, na parte debaixo, um outro quadrado, obtendo um retângulo de lados 3 e 5 . Agora, é só repetirmos o procedimento e formarmos outros quadrados de lados 5, 8, 13, 21, e assim sucessivamente, onde cada lado do quadrado é igual ao maior lado do retângulo anterior. Veja na figura abaixo: 


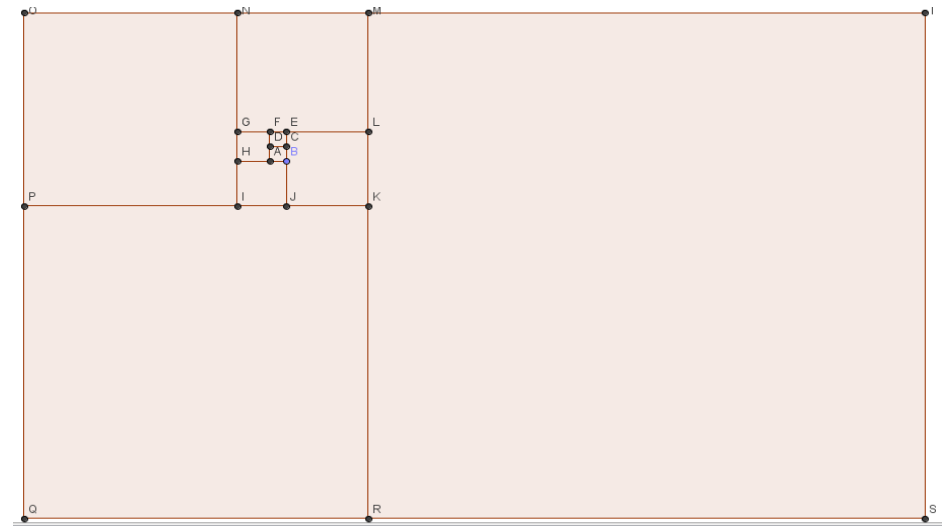

Figura 3: Retãngulo áureo Fonte: feita pelo autor

Agora, com o auxílio de um compasso, trace um quarto de círculo nos quadrados de lados: $l=21, l=13, l=8, l=5, l=3, l=2 e l=1$, obtendo a espiral áurea, como ilustra a figura abaixo:

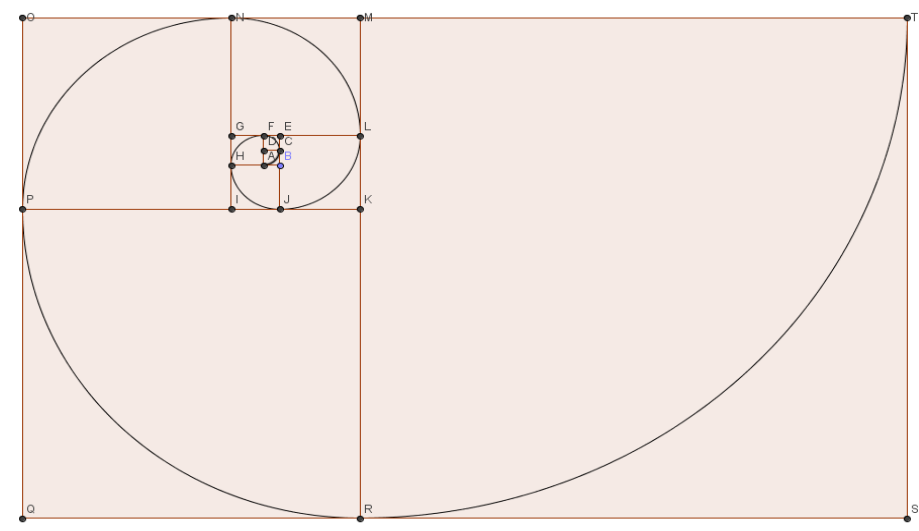

Figura 4: Espiral Áurea

Fonte: feita pelo autor

\subsubsection{A relação entre o número de ouro e a sequência de Fibonacci}

Proposição: A sequência formada pelos quocientes entre os números de Fibonacci converge para o número de ouro, ou seja, se $r_{n}=\frac{F_{n+1}}{F_{n}}, n \geq 2$ então $\lim _{n \rightarrow \infty} r_{n}=\emptyset=\frac{1+\sqrt{5}}{2}=$ $1,618033988749895 \ldots$

\section{Prova:}

De $r_{n}=\frac{F_{n+1}}{F_{n}}$ temos que:

$r_{n}=\frac{F_{n+1}}{F_{n}}=\frac{F_{n+F_{n-1}}}{F_{n}}=1+\frac{F_{n-1}}{F_{n}}=1+\frac{1}{r_{n-1}}$.

Tomando o limite em ambos os lados, segue-se que:

$\lim _{n \rightarrow \infty} r_{n}=\lim _{n \rightarrow \infty}\left(1+\frac{1}{r_{n-1}}\right)=1+\frac{1}{\lim _{n \rightarrow \infty} r_{n-1}}$ 
Seja $\lim _{n \rightarrow \infty} r_{n}=L$. Notemos que $\lim _{n \rightarrow \infty} r_{n-1}$ também vale $L$. Assim:

$L=1+\frac{1}{L} \rightarrow L^{2}-L-1=0 \rightarrow L=\frac{1 \pm \sqrt{5}}{2}$

Como $\lim _{n \rightarrow \infty} r_{n}>0 \rightarrow L=\frac{1+\sqrt{5}}{2}=\varnothing$, como queríamos provar.

\section{A sequência de Fibonacci e o mundo material}

Nesta seção iremos apresentar algumas aplicações da sequência de Fibonacci na natureza e no mundo material.

\subsection{A sequência de Fibonacci na natureza}

A sequência de Fibonacci está intimamente relacionada com a natureza. Ela aparece em inúmeras situações, seja na forma de sequência numérica ou através da espiral de Fibonacci, como por exemplo: nos troncos de árvores, em folhas, frutos, animais, etc. Nas figuras abaixo temos a concha do nautilus marinho e uma planta que descrevem, respectivamente, a espiral áurea e os números de Fibonacci. Também temos a aparição dos números de Fibonacci nas espirais do girassol (21 no senti anti-horário e 34 no sentido horário) e no número de pétalas da margarida (34 pétalas).
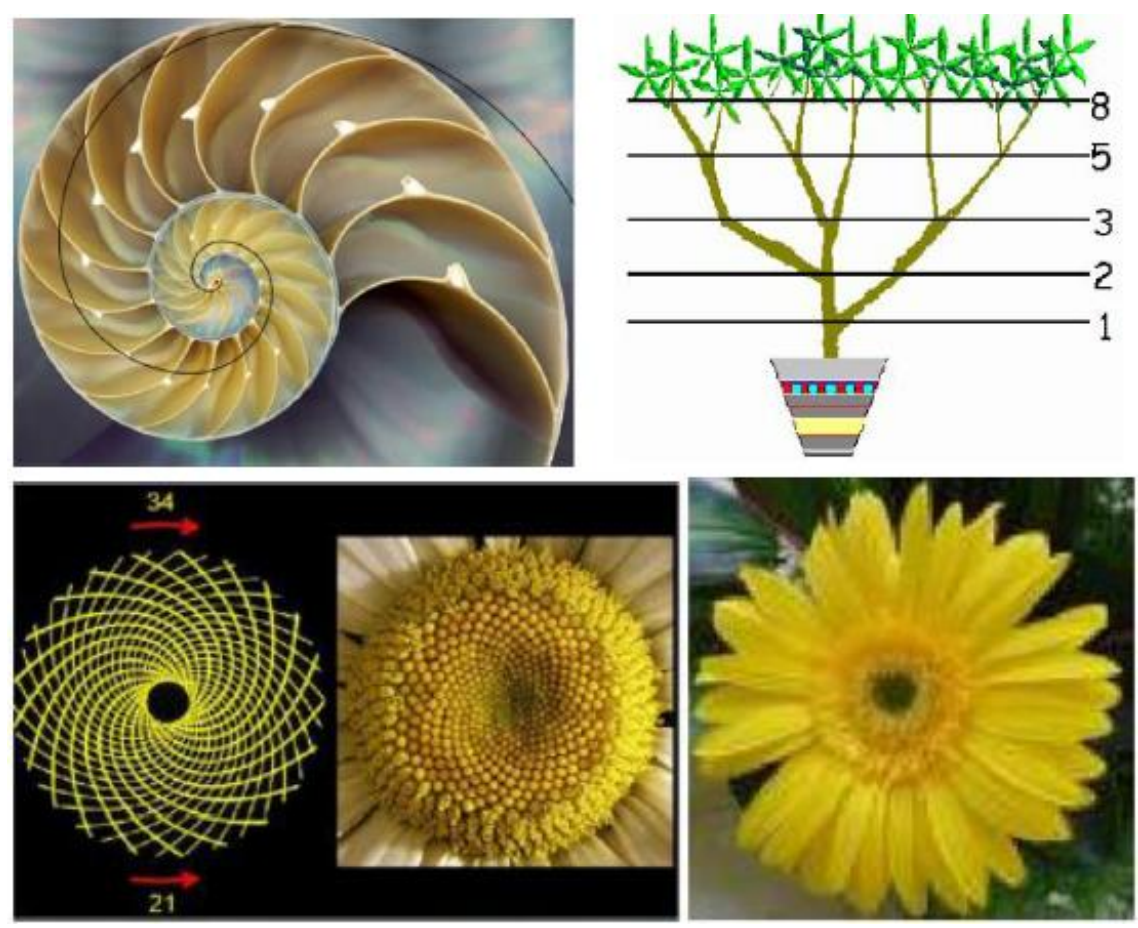

Figura 5: A sequência de Fibonacci na natureza

Fonte: Belini (2015, pg. 46 e 26) e Leopoldino (2016,pg. 21) 
Bem, estes foram alguns exemplos de observações da sequência de Fibonacci na natureza. No entanto, estas observações não param por aí. Existem muitas outras situações em que podemos perceber a mesma.

Os números de Fibonacci aparecem em muitas situações práticas. Abaixo, faremos a exposição de algumas dessas aplicações.

\subsection{Conversão de milhas para quilômetros}

Uma milha é uma unidade de medida que equivale a 1609 metros, ou seja, 1,609 quilômetros. Note que este número é bem próximo do número de ouro cujo valor é $1,618 \ldots$ Assim, por exemplo, para converter 5 milhas em quilômetros, basta olhar para o próximo número de Fibonacci depois do 5, que é o 8, pois como sabemos o número 5 é um número de Fibonacci. Caso o número desejado não seja um número da sequência de Fibonacci, multiplicamos este número pela aproximação do número de ouro 1,61, obtendo se assim um bom resultado de conversão.

\subsection{A sequência de Fibonacci na Física}

$\mathrm{Na}$ óptica dos raios de luz podemos verificar a presença da sequência de Fibonacci. Vamos considerar duas placas de vidro, de índices de refração diferentes, justapostas uma sobre a outra. Sabemos que um raio de luz que incida sobre esse conjunto pode sofrer reflexões e desvios. Assim sendo, vamos contar o número de caminhos possíveis de um raio de luz aumentando gradualmente o número de reflexões nesses caminhos.

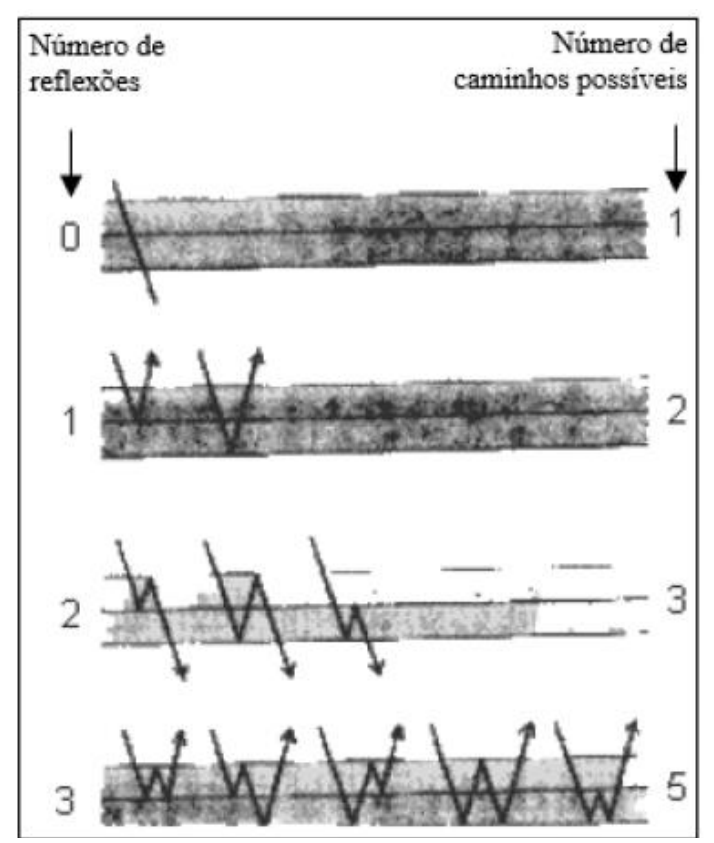

Figura 6: Relexão da luz

Fonte: Barbosa (2017,pg. 46) 


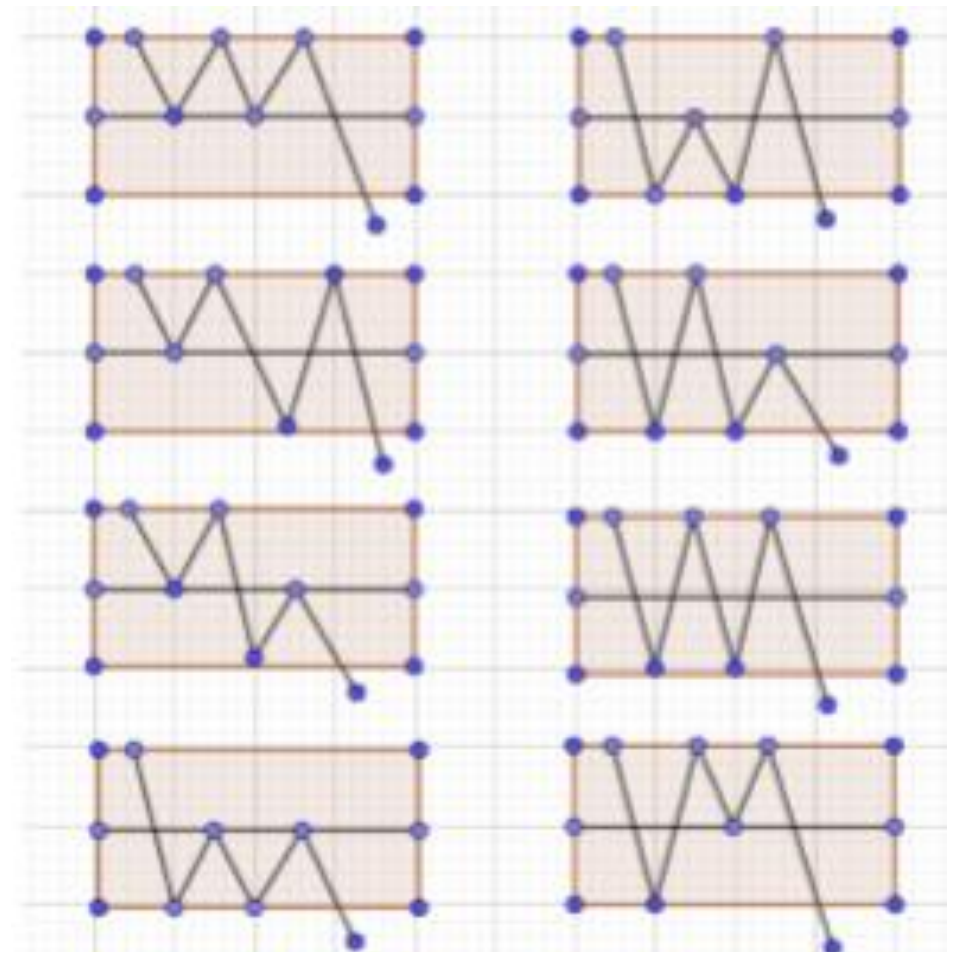

Figura 7: Número de caminhos da luz com 4 reflexões Fonte: feita pelo autor

Denotando por $f(n)$ o número de caminhos possíveis percorridos pelo raio de luz, onde $n$ é o número de reflexões, temos que:

$f(n)=F_{n+2}$

Daí:

$$
\begin{aligned}
& f(0)=F_{2}=1 \\
& f(1)=F_{3}=2 \\
& f(2)=F_{4}=3 \\
& f(3)=F_{5}=5 \\
& f(4)=F_{6}=8
\end{aligned}
$$

\subsection{A sequência de Fibonacci e o triângulo de Pascal}

O triângulo de Pascal, em homenagem ao matemático Blaise Pascal, é um triângulo numérico infinito formado por números binomiais $\left(\begin{array}{l}n \\ k\end{array}\right)$, onde $n$ representa o número da linha (posição horizontal) e $k$ representa o número da coluna (posição vertical), iniciando a contagem a partir do zero. Veja a figura abaixo: 


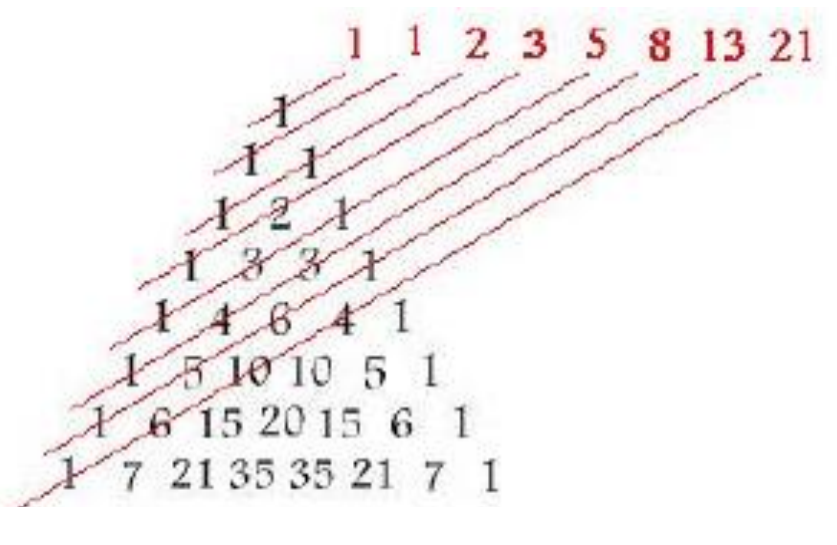

Figura 8: Triâgulo de Pascal

Fonte: Leopoldino (2016, pg. 17)

Olhando a soma das diagonais do triângulo acima, podemos ver facilmente que cada soma é um número da sequência de Fibonacci.

\subsection{A sequência de Fibonacci e o teorema de Pitágoras}

A sequência de Fibonacci está intimamente relacionada com o teorema de Pitágoras. Essa relação é estabelecida pela proposição abaixo:

Proposição: A soma dos quadrados de dois números consecutivos da sequencia de Fibonacci é um número de Fibonacci, ou seja, $F_{2 n+1}=\left(F_{n}\right)^{2}+\left(F_{n+1}\right)^{2}, \forall n \geq 1$.

\section{Prova:}

Para mostrarmos esta relação, iremos primeiro demonstrar a seguinte propriedade dos números de Fibonacci: $F_{m+n}=F_{m-1} F_{n}+F_{n+1} F_{m}, \forall n, m \geq 1$.

Faremos a prova usando indução matemática sobre $n$.

Para $n=1$, temos que: $F_{m+1}=F_{m-1}+F_{m}=F_{m-1} F_{1}+F_{2} F_{m}$. Logo o caso base é verdade.

Supondo agora que para algum $n \geq 1, F_{m+n}=F_{m-1} F_{n}+F_{n+1} F_{m}$, mostraremos que $F_{m+n+1}=F_{m-1} F_{n+1}+F_{n+2} F_{m}$.

Temos que:

$F_{m+n+1}=F_{m+n}+F_{m+n-1}=F_{m-1} F_{n}+F_{n+1} F_{m}+F_{m+n-1}=F_{m-1} F_{n}+F_{n+1} F_{m}+$ $F_{m-1} F_{n-1}+F_{n} F_{m}=F_{m-1}\left(F_{n}+F_{n-1}\right)+F_{m}\left(F_{n+1}+F_{n}\right)=F_{m-1} F_{n+1}+F_{n+2} F_{m}$, como queríamos provar.

Assim, tomando $m=n+1$, temos que:

$F_{2 n+1}=\left(F_{n}\right)^{2}+\left(F_{n+1}\right)^{2}, \forall n \geq 1$, provando assim a proposição.

\subsection{Arte e arquitetura}

$\mathrm{Na}$ arte, muitos artistas consagrados, como Piet Mondrian, Cândido Portinari, Michelangelo, Leonardo da Vinci, usaram a razão áurea em suas obras artísticas, com o intuito de obter harmonia, beleza e perfeição. Como exemplo, podemos citar a famosa pintura Monalisa do famoso pintor italiano Leonardo da Vinci, produzido em 1505. Nesta obra, há a aparição de vários retângulos áureos, como por exemplo, em torno do rosto, num retângulo de dimensões $4,1 \mathrm{~cm}$ por 2,533 cm, cuja razão é de aproximadamente 1,618. 
$\mathrm{Na}$ arquitetura, com o mesmo objetivo de obter harmonia, beleza e perfeição, muitos arquitetos usaram em suas construções o número de ouro. Um dos exemplos mais ilustres é o Partenon, na Grécia, que foi obra do Grego Phídias, que viveu em 490-431 a.C. e foi o escultor também da estátua de Atena e de Zeus e era considerado um dos mais brilhantes arquitetos da Grécia Antiga. Nesta obra, percebe-se inúmeras aparições do retângulo áureo em sua estrutura.
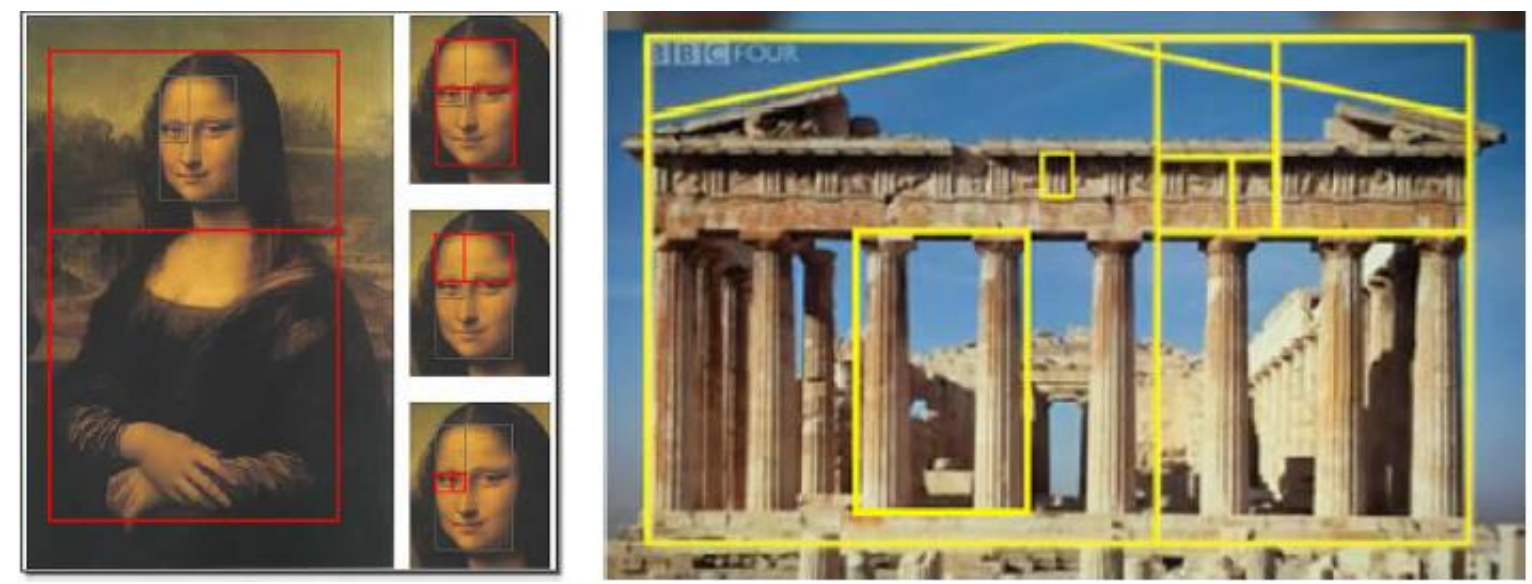

Figura 9: A Monalisa e o Partenon

Fonte: Belini (2015,pg. 24) e Barbosa (2017,pg. 68)

\section{Conclusão}

Como vimos, a sequência de Fibonacci foi oriunda do problema de Fibonacci de reprodução de coelhos e que a mesma é muito rica em propriedades interessantes. Também conhecemos o número de ouro e evidenciamos o retângulo e a espiral áurea, obtendo sua construção. Mostramos também a notável conexão entre a sequência e o número de ouro. Por último, vimos várias aplicações da mesma no mundo material, mostrando que ela está intimamente relacionada em muitas situações práticas. Dessa forma, por tudo que foi exposto, podemos concluir que este trabalho foi de extrema relevância, pois possibilitou um conhecimento muito rico sobre a sequência de Fibonacci e o número de ouro, bem como sua relação e propriedades, mostrando várias aplicações interessantes no mundo material. Esperamos que ele seja fonte de pesquisa para muitas pessoas que queiram conhecer tal sequência e que este conteúdo venha a ser trabalhado em sala de aula, devido a sua vasta riqueza.

\section{Referências}

BARBOSA, F. A. Proposta de abordagem da Sequência de Fibonacci e razão áurea no ensino médio: teoria e aplicações. 2017. 83 f. Dissertação (Mestrado) - Programa de Mestrado Profissional em Matemática, Instituto de Ciências Exatas, Universidade de Brasília, Brasília, 2017.

BELINI, M. M. A razão áurea e a sequência de Fibonacci. 2015. 67 f. Dissertação (Mestrado em Ciências) - Programa de Mestrado Profissional em Matemática, Instituto de Ciências Matemáticas e Computação, Universidade de São Paulo, São Carlos, 2015. 
BOYER, C. B. História da matemática. Tradução: Elza F. Gomide. São Paulo: Edgard Blucher, 1974.

CONTADOR, P. R. M. A matemática na arte e na vida. 2. ed. rev. São Paulo: Livraria da Física, 2011.

EVES, H. Introdução a história da matemática. Tradução: Hygino H. Domingues.Campinas: Editora UNICAMP, 2004.

LEOPOLDINO, K. S. M. Sequências de Fibonacci e razão áurea: aplicações no Ensino Básico. 2016. 103 f. Dissertação (Mestrado) - Programa de Mestrado Profissional em Matemática, Centro de Ciências Exatas e da Terra. Universidade Federal do Rio Grande do Norte, Natal, 2016.

OLIVEIRA, K. I. M.; FERNÁNDEZ, A. J. C. Iniciação á matemática: um curso com problemas e soluções. 2. ed. Rio de Janeiro: SBM, 2010.

SILVA, R. R. Razão áurea como motivação ao estudo de conteúdos matemáticos. 2014. 114 f. Dissertação (Mestrado em Matemática) - Programa de Mestrado Profissional em Matemática, Universidade Federal de Goiás, Catalão, 2014.

VOROBIOV, N. N. Números de Fibonacci. Tradução: Carlos Vega. Moscou: MIR, 1974. (Lecciones populares de matemáticas).

ZAHN, M. Sequência de Fibonacci e o número de ouro. Rio de Janeiro: Ciência. Moderna, 2011. 